INPLASY

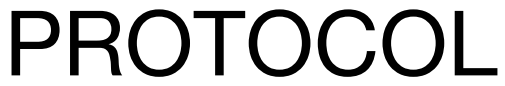

To cite: Huang et al.

Prognostic value of net water uptake in acute ischemic stroke: a protocol for a systematic review and metaanalysis. Inplasy protocol 2021120077. doi:

10.37766/inplasy2021.12.0077

Received: 17 December 2021

Published: 17 December 2021

Corresponding author:

Wanqian Liu

wqliu@cqu.edu.cn

Author Affiliation:

Bioengineering College,

Chongqing University.

Support: None.

Review Stage at time of this submission: The review has not yet started.

Conflicts of interest:

None declared.

\section{Prognostic value of net water uptake in acute ischemic stroke: a protocol for a systematic review and meta-analysis}

Huang, W1; Yang, D2; Fan, D3; Hou, C4; Liu, W5.

Review question / Objective: The purpose of this protocol is to present a transparent and clear methodology for performing a systematic review and meta-analysis of the available literature aimed to answer the following question: among patients with acute ischemic stroke due to large vessel occlusion, is net water uptake (NWU), as measured in CT images, associated with (i) cerebrovascular complications, including malignant cerebral edema, secondary intracerebral hemorrhage, and (ii) post-stroke functional outcome as measured by the modified Rankin Scale.

Condition being studied: Currently, the association between net water uptake with cerebrovascular complications or poststroke functional outcomes is not well defined. Broocks found that NWU based on CT was an important marker for malignant edema in LVO patients and independently associated with clinical prognosis. Additionally, Nawabi indicated that a higher degree of NWU was a predictor of intracranial hemorrhage in patients with acute ischemic stroke treated with mechanical thrombectomy. Thus, a higher NWU might contribute to the development of stroke complications and poorer outcomes. However, no systematic review and meta-analysis to quantitatively summarize this evidence and help establish the predictive value of NWU in patients with acute ischemic stroke.

INPLASY registration number: This protocol was registered with the International Platform of Registered Systematic Review and Meta-Analysis Protocols (INPLASY) on 17 December 2021 and was last updated on 17 December 2021 (registration number INPLASY2021120077).

\section{INTRODUCTION}

Review question / Objective: The purpose of this protocol is to present a transparent and clear methodology for performing a systematic review and meta-analysis of the available literature aimed to answer the following question: among patients with acute ischemic stroke due to large vessel occlusion, is net water uptake (NWU), as 
measured in CT images, associated with (i) cerebrovascular complications, including malignant cerebral edema, secondary intracerebral hemorrhage, and (ii) poststroke functional outcome as measured by the modified Rankin Scale.

Rationale: Worldwide, stroke is the second most common cause of mortality and the second most common cause of disability. Large hemispheric infarction is a most severe subtype of acute ischemic stroke which due to large vessel occlusion (LVO), often leads to severe disability and mortality by progressive cerebral edema. Despite the timely endovascular treatment, the prognosis of nearly half of the patients remains poor. Exploring the novel biomarkers for the prediction of the malignant cerebral edema and prognosis of patients with LVO is therefore urgently needed so that tailored treatment strategies could be developed to improve clinical efficacy.

Condition being studied: Currently, the association between net water uptake with cerebrovascular complications or poststroke functional outcomes is not well defined. Broocks found that NWU based on CT was an important marker for malignant edema in LVO patients and independently associated with clinical prognosis. Additionally, Nawabi indicated that a higher degree of NWU was a predictor of intracranial hemorrhage in patients with acute ischemic stroke treated with mechanical thrombectomy. Thus, a higher NWU might contribute to the development of stroke complications and poorer outcomes. However, no systematic review and meta-analysis to quantitatively summarize this evidence and help establish the predictive value of NWU in patients with acute ischemic stroke.

\section{METHODS}

Search strategy: PubMed search terms: \#1 cerebrovascular disorders [Mesh] | \#2 stroke* OR apoplex* OR cerebral vasc* OR

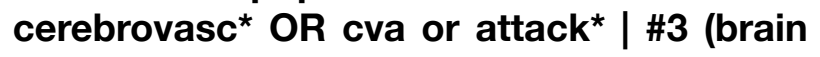
OR cerebr* OR cerebell* OR vertebrobasil ${ }^{\star}$ OR hemispher* OR intracran* OR intracerebral OR infratentorial OR supratentorial OR middle cerebr* OR mca* OR "anterior circulation") AND (ischemi* OR ischaemi* OR infarct* OR thrombo* OR emboli* OR occlus* OR hypoxi* OR apoplexy) | \#4 \#1 OR \#2 OR \#3 | \#5 "Water/ metabolism"[Mesh] | \#6 "water uptake" OR NWU | \#7 \#5 OR \#6 | \#8 Animals NOT Humans | \#9 \#4 AND \#7 | \#10 \#9 NOT \#8.

Participant or population: Inclusion: (i) patients were diagnosed with acute ischemic stroke by CT or magnetic resonance imaging; (ii)during admission, net water uptake was assessed or calculated; (iii) population of adults aged 18 or older with any restriction on the race or gender.

Intervention: The comparison should be made between patients in the upper range of net water uptake (exposed) and those in the lower range of net water uptake. There is no established net water uptake threshold for acuteischemic stroke. The upper and lower ranges of net water uptake will likely vary in the researches included in our systematic review.

Comparator: The comparison should be made between patients in the upper range of net water uptake (exposed) and those in the lower range of net water uptake. There is no established net water uptake threshold for acuteischemic stroke. The upper and lower ranges of net water uptake will likely vary in the researches included in our systematic review.

Study designs to be included: Prospective or retrospective observation studies were considered eligible.

Eligibility criteria: Prospective or retrospective observation studies were considered eligible.

Information sources: The literature search will be performed using MEDLINE (via PubMed), Web of Science, EMBASE, and Cochrane Central Register of Controlled Trials (CENTRAL) database by applying predefined search terms from the date of inception of the database to December 
2021. In addition, the references lists of the retrieved articles were also manually searched to identify relevant articles.

Main outcome(s): Primary outcome: cerebrovascular complications, which included malignant cerebral edema and secondary intracerebral hemorrhage during hospitalization which were defined by each article.

Additional outcome(s): Secondary outcome: post-stroke functional independence. Good functional outcome is defined as modified Rankin Scale (mRS) of 0-2 points and poor functional outcome is defined as modified Rankin Scale (mRS) of 3-6 points.

Quality assessment / Risk of bias analysis: To assess the methodological quality and risk of bias of each literature included, we will use The Newcastle Ottawa Quality Assessment Scale: Cohort Studies (NOS) which was recommended by Cochrane non-randomized study method group in three parts: selection, comparability, and exposure.

Strategy of data synthesis: We will employ STATA 16.0 to synthesize and analyze data. All continuous data will be expressed as mean difference (MD) or standardized mean difference (SMD) and $95 \%$ confidence intervals, while all dichotomous data will be calculated as risk ratio or odds ratio and $95 \%$ confidence intervals. The heterogeneity was quantified with the $\mathbf{I}^{2}$ statistic, and $25,50,75 \%$ are considered to represent low, medium, high heterogeneity, respectively. When there was moderate and high heterogeneity in the study, the random-effects model was employed for analysis based on the DerSimonian-Laird model. In addition, the presence of publication bias will be assessed by using an enhanced funnel plot, Duval and Tweedie's trim and fill method and Egger's regression intercept if at least 10 studies are retrieved.

Subgroup analysis: Subgroup analyses will be performed based on the age of the study participants, sample size, location, and NOS score to detect sources of heterogeneity and observe whether the results are credible.

Sensitivity analysis: We will use the "leave one out" method as a sensitivity analysis to test the responsible studies of heterogeneity and identify the robustness of study findings.

Language: English language studies.

Country(ies) involved: China.

Keywords: net water uptake; stroke; large vessel occlusion.

Dissemination plans: We will publish the review on completion.

Contributions of each author:

Author 1 - Wei Huang - W.H. conceived and designed the protocol. Additionally, W.H. is responsible for searching the data, conducting the systematic review and meta-analysis, and drafting the manuscript. Email: huangweicn@cqu.edu.cn

Author 2 - Dahong Yang - D.Y. is responsible for searching the data and extracting the data.

Email: ydhajy1993@126.com

Author 3 - Danyang Fan - D.F. is responsible for extracting the data and conducting the systematic review and meta-analysis.

Email: 1219901996@qq.com

Author 4 - Chao Hou - C.H. is responsible for conducting the systematic review and meta-analysis.

Email: houchaotd@163.com

Author 5 - Wanqian Liu - W.L. conceived and designed the protocol. Additionally, W.L. is responsible for drafting the manuscript.

Email: wqliu@cqu.edu.cn 